\title{
CARMEN: Delivering Carrier Grade Services over Wireless Mesh Networks
}

\author{
Invited Paper
}

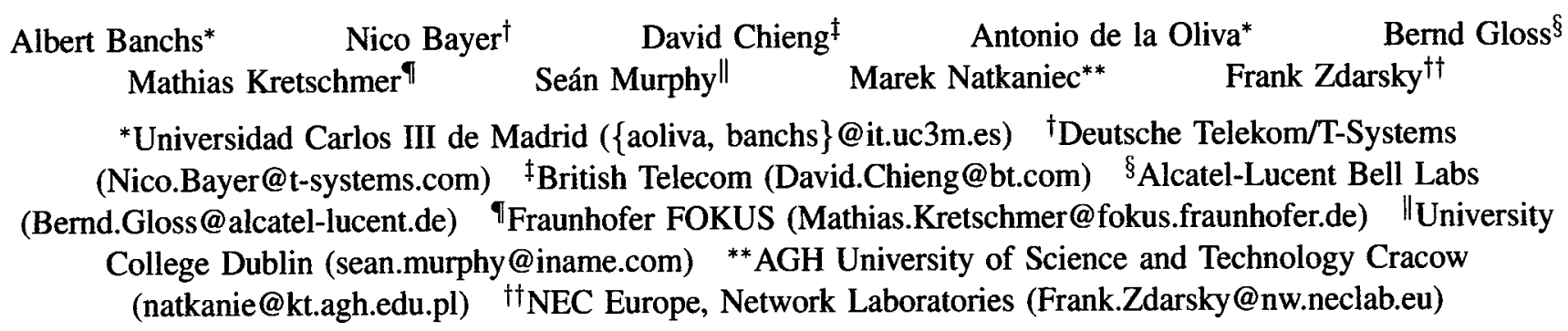

\begin{abstract}
Current Internet use is evolving, users are becoming mobile and are expecting data services on the go. This fact presents big challenges and opportunities to operators, which see the increase in data services as a big market still to be exploited. However, current cellular technologies cannot accommodate the demand that will arise when the true Mobile Internet evolves. Addressing these challenges, we present the CARMEN project, the vision of which is to extend operators' infrastructure by providing carrier grade services through a heterogeneous wireless mesh. The CARMEN architecture will provide enough bandwidth to cope with users' expectations at a reduced cost, thereby generating major benefits to operators and users.
\end{abstract}

\section{INTRODUCTION}

The need for wireless communications anytime anywhere is almost upon us. In the last few years, the amount of data traffic on wireless networks has increased dramatically and users are becoming increasingly comfortable with being able to access data services on the move. However, most believe that wireless communications is still in its infancy. A wider range of innovative and bandwidth-intensive applications will emerge and therefore fuel a greater need for bandwidth. Providing access to data services for large amounts of mobile users, however, is a costly endeavour. Current cellular based access systems cannot accommodate the demand that will arise when the true Mobile Internet evolves. Hence, there is a need to consider alternative solutions which can offer lower cost wireless network access. One candidate solution is that of mesh networks. That is the focus of this work. Mesh networking technology provides a cost effective and efficient alternative for realising backhaul networks to provide mobile users with potentially high quality services. The multihop wireless network architecture of mesh networks enables them to efficiently cover large areas without requiring many interconnections into a wired infrastructure. Further, mesh

This work was partially funded by the European Commission within the 7th Framework Program in the context of the ICT project CARMEN [1] (Grand Agreement No. 214994). The views and conclusions contained here are those of the authors and should not be interpreted as necessarily representing the official policies or endorsements, either expressed or implied, of the CARMEN project or the European Commission. networks are dynamically self-organised and self-configured, which ultimately results in reduced up-front cost $(\mathrm{CapEx})$ and lower network maintenance costs (OpEx) for the operator. A critical concern for operators is to provide access to the typical service bundle via all the radio access networks. Specifically, access to typical voice, video and data services must be provided. This imposes some constraints on the performance of the mesh backhaul: it must support carrier grade service provisioning. Carrier grade, in general, has many aspects, including equipment reliability, security, AAA, QoS, management, standards compliance, mobility support, service integration, etc; To achieve carrier grade capacity while preserving flexibility and cost efficiency is a great challenge due to wireless mesh inherent limitations namely throughput and scalability. In order to have a better insight on how to design a carrier grade network, many major operators have already considered wireless mesh networks (WMNs) as a technology for their wireless Cities initiatives [2] [3] [4] [5]. However, they have yet to fulfill the carrier-grade attribute as these deployments are still heavily dependent on $\mathrm{WiFi}[6]$ as the underlying radio technology. Envisioning these challenges, the CARMEN (CARrier grade MEsh Networks) project, funded by the European Seventh Framework Program, was instigated. This project joins operators (British Telecommunications, Deutsche Telekom), manufacturers (Alcatel-Lucent, NEC) and research organizations (Akademia Gorniczo-Hutnicza, Fraunhofer Institute, University College Dublin and University Carlos III de Madrid) in the search of a mesh architecture which will focus on those aspects of carrier grade most related to reliable service delivery: specifically, on issues relating to ensure that typical carrier service offerings, such as voice, video and data, are delivered with appropriate service quality to diverse mobile devices. This is a real challenge which is not supported by current mesh network technology. The promises of WMNs have triggered advances at various levels. Firstly, vendors are pushing their proprietary mesh products. For example 
BelAir $^{1}$, Cisco $^{2}$ and Strix Systems ${ }^{3}$ are offering mesh solutions for building automation, small-scale and large-scale Internet connectivity, and so on using proprietary equipment. Secondly, community mesh networks grow to provide connectivity and capacity. The MIT Roofnet ${ }^{4}$ or the Freifunk in Berlin ${ }^{5}$ are examples that have grown to a size of up to 200 access points and are continuing to increase. Thirdly, standardization activities are focusing on multi-hop and mesh networks. Two of the most compelling technologies for which standardized multi-hop mesh/relay variants are currently under development are WiFi [7] and WiMAX [8]. Both of these can be combined to realize a heterogeneous mesh backhaul solution even if these technologies have different and complementary characteristics-range, cost, etc. CARMEN will focus on the delivery of carrier grade services in the context of heterogeneous wireless mesh networks. The project results will provide a network concept with the following benefits over current deployments: i) Carrier grade service support ii) Link and technology abstraction to enable heterogeneous technologies iii) Quality of Service (QoS) and mobility support and iv) Ease of deployment and operation due to self configuration and continuous monitoring.

The structure of the paper is as follows: Section II studies different scenarios where the deployment of a wireless mesh network provides extended benefits. Based on these scenarios a set of functional requirements are presented in section III. The proposed architecture of the CARMEN network is shown in section IV, with detailed explanation of the overall architecture in section IV-A, main functionality in section IV-B and the node architecture in section IV-C. Finally we conclude in section $\mathrm{V}$.

\section{SCENARIOS}

In order to develop an appropriate solution for delivery of carrier grade services over mesh networks, it is useful to start with typical scenarios which can be used as an input to the requirements specification process. Hence, first activities of the project are mainly focused on identifying realistic scenarios for wireless mesh networks. Two basic scenarios underpin the work of the project: a city coverage scenario and an emergency scenario. It is obvious that both scenarios are very different in nature and also have vastly different requirements. The goal of CARMEN is to design an architecture that may be parameterized for efficient operation in both "extreme cases".

\section{A. City Coverage Scenario}

In the city coverage scenario, wireless mesh networks are deployed across some selected areas of the city centre to provide users access to operator's services. In this scenario the mesh technology presents a cost effective alternative to traditional technologies as it allows for a flexible and fast network deployment without the need to connect every access

\footnotetext{
lhttp://www.belairnetworks.com/

2http://Www.cisco.com/

${ }^{3}$ http: //www.strixsystems.com/

${ }^{4}$ http://pdos.lcs.mit.edu/roofnet/

5 http://freifunk. net/
}

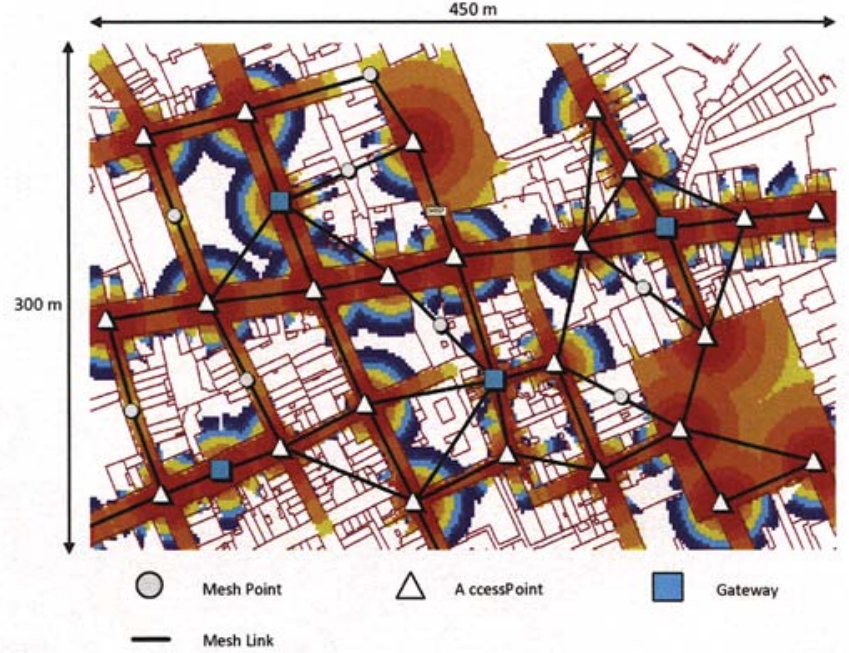

Fig. 1. An Outdoor Scenario in Central West London

point or base station via a wired link with the core network. The mesh network comprises of a number of nodes which are connected into the operator's wired infrastructure and a larger number of nodes which only have wireless connectivity. End-user traffic, then, is typically routed to/from the wired infrastructure through the wireless mesh from/to the end-user terminal. Fig. 1 depicts a relatively dense outdoor deployment scenario covering $0.135 \mathrm{~km}^{2}$ area in central west London. The set of services and the user base that the mesh must support can differ from one deployment to the next. Typical services would include

- Basic Internet access (non delay sensitive)

- Contiguous wireless coverage to enable delay sensitive applications such as voice. This requires a coverage solution which provides mobility support.

- Virtual private network (VPN) services.

- Wireless closed circuit television (CCTV) to aid community safety and security.

- Distribution of broadcast and/or localized video services to, for example, handsets and/or video screens within the mesh coverage area.

The user base would vary from city to city and would depend on the population density and/or the density of users in the urban centre at peak times during the day. The set of services and the user base drive the demands of the network. These are used to determine the complex, coupled characteristics of the mesh which include the following:

- data rate per user

- density of gateways/interconnects between the mesh and the wired infrastructure

- density of nodes in the mesh

- radio technologies used for interconnection of the different nodes

- delay constraints on traffic

- end user terminal speed to be supported

- size of the network (coverage area, number of nodes)

The CARMEN project has been working on defining scenarios which provide a realistic backdrop for the work. However, 
not all of the details have been defined as yet; in particular, some aspects of the service mix and the resulting demands per user have not been defined. However, the project has decided to focus on larger city coverage problems-with a $100 \mathrm{~km}^{2}$ coverage area target ${ }^{6}$ and a set of users, most of whom have low mobility rates, typically walking speed.

\section{B. Emergency Scenario}

The emergency scenario is one in which a wireless mesh network needs to be rapidly deployed in a disaster area in which any communication infrastructure has been totally destroyed. In this case a mesh network architecture provides the ability for an easy and rapid radio access coverage that is used for coordination of relief workers as ambulance, volunteers and rescue teams. The emergency mesh network is connected via at least two independently operation gateways to the backbone (either via satellite to a remote internet service provider or via terrestrial fixed or wireless lines to the existing conventional communication network). The deployment of the emergency mesh networks can be divided into different phases. In an early phase the network is considered to provide a basic communication infrastructure for rescue teams and traffic flows are mainly intra-mesh. Focus in these early stages is on coverage and reliability. It is expected that only low capacity with poor/intermitted connectivity to the core is provided. In later phases the performance of the network is increased stepwise and the amount of Internet traffic increases as the access to the network is granted to other user groups, e.g., refugees and victims. Services would include i) Point-to-Point and Point-to-Multipoint communication, ii) Low data rates, e.g., 4.8-28 kb/s for TETRA[9] messaging data/voice, iii) High date rates for video applications, iv) Basic Internet access is provided at later stages.

These services raise the following network demands:

- Service prioritization to distinguish at least between high priority (signaling, management) and other traffic.

- Coverage is the most important requirement, especially in early stages and service quality is not as important as in the city coverage scenario. Even higher delays are acceptable

\section{FunCTIONAL REQUiREMENTS}

In this section we outline the Functional Requirements to be fulfilled by a CARMEN mesh which have been derived from the scenario specifications. Today's carriers need to address customer demands for a number of services requiring more than best effort network performance. In parallel, the demand for resources increases steadily and reaches temporary peaks during special events (sports, disaster, etc.). To address those requirements, a CARMEN mesh aims at providing differentiated QoS guarantees, supporting the use of complementary wireless technologies as well as exploiting the advantages of self-management techniques to keep the deployment and maintenance costs low. CARMEN's multihop nature allows for new nodes to easily be added increasing

\footnotetext{
${ }^{6}$ The area of Paris is $86 \mathrm{~km}^{2}$
}

the coverage area or the available resources. To efficiently address the requirements of a carrier-grade heterogeneous multi-technology mesh, CARMEN introduces functions performing complementary tasks such as QoS-aware routing and forwarding, user mobility, integration of complementary wireless technologies, network planning and radio resource management and monitoring and self-configuration.

Robust QoS resource management is a crucial requirement to be fulfilled by a CARMEN mesh in order to smartly control resources allocation and policing, thus avoiding shortages due to (required) resource usage exceeding the available capacity. This requirement derives from carrier demands to support triple-play services over a heterogeneous wireless mesh network which also implies an admission control mechanism combined with a resource reservation mechanism in order to satisfy the requested bandwidth and latency requirements. To keep the management overhead low, all traffic is classified into one of four possible classes with traffic within the same traffic class being treated equally inside the CARMEN mesh. Multiple flows from one Gateway to User Terminals (UTs) behind the same Access Point (AP) belonging to the same traffic class are being treated as aggregates. Taking into account the often asymmetric and even uni-directional nature of wireless links and to exploit available resources efficiently, CARMEN treats links as uni-directional which also allows CARMEN to perform load balancing, etc. by using multipath routing. Addressing the QoS requirements of the various operator services, CARMEN computes a path through the mesh network that matches the requested QoS requirements as specified by the resource management module. CARMEN paths typically connect an AP to a Gateway or, as an optimization, might link two APs directly for inter-mesh communication. In order to simplify NetLMM ${ }^{7}$-like UT mobility, CARMEN does not configure paths directly to an UT. Instead, paths are terminated at the APs which act as points of attachment (PoA) for UTs. Once the path is computed routing/forwarding state is updated on all intermediate nodes along the new path, where necessary. Increasing demand for triple-play service requires operators to provide high quality multi-media content to its customers. This requirement is addressed by supporting 1-to$\mathrm{N}$ Multicast distribution within the CARMEN mesh taking into account technology-specific optimizations in order to keep the channel resources allocation as low as possible. To efficiently distribute content to groups with many subscribers, CARMEN attempts to leverage the use of long range overlay cells (i.e. DVB [10] or WiMAX) to reduce the load on the hop-byhop mesh links. A CARMEN mesh network typically consists of heterogeneous but complementary radio technologies allowing an operator to choose the proper technology for a given scenario. Interface configuration is performed through an Abstract Interface allowing the higher CARMEN layers to be technology-agnostic. To ease the integration of emerging technologies into CARMEN, merely a technology abstraction wrapper is needed. Today's wireless users are highly mobile which draws a special focus to CARMEN's mobility support which is charged with maintaining link layer connectivity

\footnotetext{
${ }^{7}$ Network-based Localized Mobility Management
} 
of a given QoS between UTs and their PoA. The Mobility Management function will also trigger the re-establishment of a UT's network layer connectivity after a change of PoA which is initiated by the CARMEN mesh based on access link and mesh capacity changes. Seamless handover is supported for IEEE 802.21-capable [11] UTs. The reduction of network deployment and maintenance costs is nowadays very important for mesh operators especially when delivering carrier grade services in highly scalable networks. To fulfill this requirement the self-configuration techniques must be considered. The ability for dynamic self-configuration is one of the fundamental CARMEN function because it allows to simplify installation, administration, and management of the network. It can also provide greater flexibility and reduce the operational costs. As a consequence of that all mesh nodes have to be selfadaptive with regard to their initial configuration, including the update of neighbor nodes, and support dynamic reconfiguration and compensation in failure cases. All these procedures are performed continuously, from initial deployment to regular operation modes of the network. It is essential for a newly introduced mesh node to intelligently detect neighbors and their capabilities. The bootstrap phase uses self-configuration techniques to discover the initial network topology. This process creates a logic topology map of the initial network infrastructure, which is provided to the nodes routing module as initial basis for its operation. Radio frequency spectrum is a scarce limited natural resource. That's why the ability to use the available spectrum in an efficient manner is so crucial. In regular operation phase the self-configuration function takes care of frequency management and cross-layer-aware radio. The implementation of this function will also involve enhanced monitoring techniques. The important characteristic of carrier grade mesh is to ensure $24 / 7$ smooth operation of the network. The monitoring system plays a critical role to support this functionality. Aggregation, correlation and statistical analysis of the gathered data on different timescales is essential for tracking and keeping up-to-date the states or information of the mesh network. The reports from the monitoring system are not only crucial for self-configuration and planning functions, but also important for the dynamic resource management, such as routing updates or QoS including admission control decisions. The maximization of network capacity (e.g., throughput, jitter, delay) in a given area is another big challenge for mesh operators. This requirement is addressed by radio network planning. This phase is done prior to deployment and is dependent on external information such as site info, service requirements, etc. The radio planning is composed of fixed planning done at several weeks time granularity and dynamic planning usually done at several days time granularity on the basis of actual traffic characteristics and required multipaths from a source to a destination. The static planning ensures base radio coverage of an urban scenario resulting in a stable network infrastructure whereas the dynamic planning ensures the radio coverage for a specific area during special events (sport events, emergencies, etc) resulting in an extension of physical deployments of network equipments. Multiple radio interfaces are utilized in order to increase the number of available resources or to avoid interferences. Off-line and on-

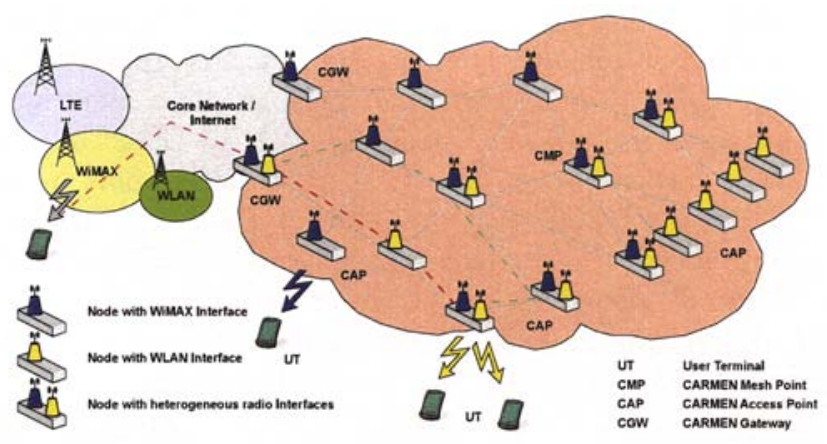

Fig. 2. Example CARMEN Network Topology

line planning tools share the task of optimally configuring and maintaining a CARMEN mesh in order to keep the management cost to the operator at a minimum.

\section{ARCHITECTURE}

This section presents the architecture of a CARMEN network with a focus on project assumptions and results from the initial architecture phase of the CARMEN project. The major aims covered by this system architecture are i) to setup a mesh topology on top of an open list of standard wireless point-tomultipoint technologies such as IEEE 802.11 and IEEE 802.16 and ii) to provide carrier-grade communication services on top of this mesh. In detail, this section comprises the topics CARMEN network topology, CARMEN mesh functions and CARMEN Mesh Point architecture.

\section{A. CARMEN Network Topology}

Fig. 2 depicts a typical CARMEN network topology introducing all types of CARMEN nodes. These are

- User Terminals (UTs)

- CARMEN Access Points (CAP)

- CARMEN Mesh Points (CMP)

- CARMEN Gateways (CGW)

Direct radio connectivity between nodes, i.e. the physical network topology, is symbolized by thin solid lines. The roles of the mentioned nodes in the CARMEN architecture are as follows:

User Terminal (UT): A UT is an end-user device which uses the CARMEN mesh to obtain access to services. It can be both a fixed or mobile device and no specific form factor is assumed. As a minimum requirement to get a basic set of services from the CARMEN mesh, User Terminals are expected to be fully compliant with the standards of the respective access technology (radio or fixed). The UTs may have advanced capabilities (e.g., IEEE 802.21 support) to avail of all of the services provided by the CARMEN mesh; alternatively, if they do not have advanced capabilities, they can still use the CARMEN mesh as an access network and obtain an acceptable level of service. End-user devices are not part of the mesh per se; they are simply devices that use the functionalities provided by the mesh.

CARMEN Mesh Point (CMP): A CARMEN Mesh Point is 
a node within the CARMEN mesh that is equipped with CARMEN capabilities related to the setup and operation of the mesh. CARMEN Mesh Points are capable of forwarding traffic using layer 2.5 or layer 3 tunneling. In forwarding the traffic, the mesh points are conscious of the requirements of the traffic, e.g., latency requirements. A CARMEN Mesh Point may have one or more radio interfaces: examples include $802.11,802.16$ and DVB interfaces.

CARMEN Access Point (CAP): A CARMEN Access Point is a CARMEN Mesh Point with the capability to provide access to the CARMEN mesh to UTs. This includes traffic handling with non-CARMEN transport options and providing terminal related functions as IEEE 802.21 handover signaling. Typically, CARMEN APs have one or more radio interfaces dedicated for use by UTs, i.e. these interfaces do not carry traffic to other CARMEN Mesh Points; however, this may not always be the case. The set of radio technologies employed on access links may be different from those used within the CARMEN mesh. For example, it is envisaged that some CARMEN APs may provide access to TETRA (TErrestrial Trunked RAdio) terminals, but the traffic may be carried over the mesh using other technologies.

CARMEN Gateway (CGW): A CARMEN Gateway is a CARMEN Mesh Point that provides connectivity to the network provider's core or backbone network: it is located at the boundary between the core network and the CARMEN mesh. The gateway has at least one standardized interface into the core network. Typically, this is a wired interface using, for example, IP and Ethernet, but it may also be a wireless connection.

Core Network/Internet: The core network is shown left in the figure and represents an IP-based infrastructure which provides connectivity between CARMEN Gateways and external entities. This is where most traffic is directed to or from. Generally in CARMEN, it is assumed that CARMEN mesh network is operated by a single network provider and has one or more connections into the provider's core network.

\section{B. CARMEN Mesh Functions}

A salient feature of the CARMEN architecture is its design for incremental deployment with low administrative overhead. The Self-Configuration function plays an important role during bootstrapping the network. After a CARMEN node is activated, the Self-Configuration function starts discovering the node's radio neighborhood as seen by each of its multiple radio interfaces of different technologies. Based on a combination of active and passive probing by the Monitoring function and additional inputs such as GPS information, the SelfConfiguration function then creates a view of the physical network topology. The physical topology describes over which radio interfaces two neighboring CARMEN Mesh Points may potentially communicate with each other and at which signal quality. Furthermore, Self-Configuration gathers information on the level of potential interference between pairs of radio links as well as with external interferers. Based on these inputs, the Network Planning and Radio Resource Management functions decide on how to allocate radio frequency spectrum and transmit power resources to each radio interface such that the resulting logical network topology is able to meet administratively specified high-level performance objectives and constraints. During the operation of the network, the Monitoring function continuously monitors the network's performance characteristics and reports any deviation to radio resource management, so that the latter can take corrective action and adapt the logical topology accordingly. To provide a carrier-grade data transport over the logical topology of the CARMEN Mesh, three architecture functions need to work hand in hand: The Routing function discovers and disseminates information about the current logical mesh topology and its QoS-related characteristics, such as available link capacities, per-hop forwarding latencies, radio interference, etc. Based on this information, it computes unicast paths and multicast trees that meet a specified QoS constraint. While the routing algorithms employed in the CARMEN architecture are designed to be aware of the wireless nature of mesh links, they are still technology agnostic, working only on the link abstraction provided by the CARMEN Abstract Interface defined below. The Routing function is able to compute multiple paths between two given CARMEN Mesh Points, which allows data traffic flows to be better distributed over the mesh topology. As a consequence, data forwarding in CARMEN is based on path identifiers rather than destination address based, which means the Routing function also needs to provide mechanisms for managing forwarding state in mesh nodes along a path. The Capacity Handling function's responsibility is to ensure the available capacity of the wireless mesh links is used efficiently while still providing the expected QoS to each traffic flow. This requires a strict flow admission control in combination with traffic policing at the ingress edge of the mesh network. Incoming user traffic is classified into the four different traffic classes supported by the CARMEN Mesh. Capacity Handling then chooses the optimal path that the classified flow should take through the mesh network to reach its destination. It also triggers the allocation of resources along each path, so that QoS characteristics of a flow remain controlled. Mobility Management has the tasks of maintaining radio link layer connectivity of a given QoS between a UT and its point of attachment to the CARMEN Mesh as well as of re-establishing the UTs' network layer connectivity after a change in point of attachment. Such a change may become necessary when the access link quality degrades below a given threshold due to movement of the UT or channel fading or when the UT requires a change of radio technology, e.g., to obtain a higher data rate. In a CARMEN mesh network context, an additional reason to change a UT's point of attachment is the need to balance the load not only over access links, but also over mesh links. To this end, Mobility Management works in close collaboration with Capacity Handling.

\section{CARMEN Abstract Interface}

One aim of CARMEN is to separate mesh functions from the specifics of a particular wireless technology. This leads to an approach of defining technology-independent CARMEN mesh functions as described above and an encapsulation and 


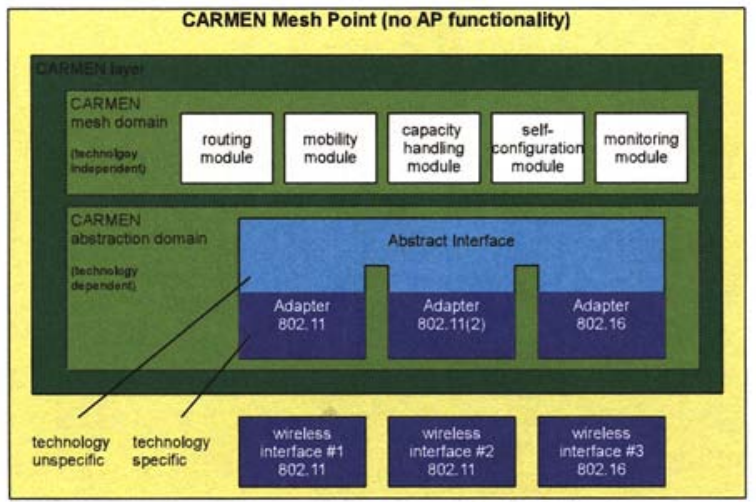

Fig. 3. Technological domains and building blocks of a CARMEN node

abstraction of specific properties, measures and API calls of wireless technologies. The interface between CARMEN mesh functions and wireless technologies is realized introducing an abstraction interface. Fig. 3 depicts the basic domains of CARMEN modules, i.e. the CARMEN mesh domain with technology independent mesh functions, and the CARMEN abstraction domain including the CARMEN Abstract Interface (AI). In first place, the architecture of CARMEN nodes is restricted to control plane issues. Data plane functions as queue handling and QoS enforcement are left as much as possible inside the technology parts. Modules realizing the CARMEN mesh functions reside inside the CARMEN mesh domain of each CARMEN Mesh Point. According to requirements identified in Section III, the modules "routing module", "mobility management module", "capacity handling module", "self-configuration module", and "monitoring module" are included. These modules realize node-specific mesh functions and inter-operate with other modules, e.g., to determine the logical CARMEN mesh topology, with centralized or decentralized coordination functions. The CARMEN abstract interface comprises (1) interface management functions, (2) basic communication mechanisms realizing service access points for CARMEN mesh functions, and (3) adapters for and extensions to wireless technologies. The abstract interface management functions are responsible for managing wireless interfaces within a CARMEN node and providing basic information about them. Basic communication mechanisms are defined according to the basic IEEE 802.21 services of "Information", "Command" and "Event". Wireless technology adapters provide mappings from technology specific API calls to CARMEN Abstract Interface calls and vice versa and mappings from technology dependent properties, measures and settings to technology independent representations, as far as this is possible and reasonable. To validate the adopted abstraction, currently two wireless point-to-multi-point communication technologies are considered for the initial design. The project aims of CARMEN include IEEE 802.11 WLAN and IEEE 802.16 WMAN technology knowing that adaptations within and outside the standards have to be considered to fulfilling the aims of a carrier-grade mesh functionality. Furthermore,
DVB technology shall be included into selected CARMEN Mesh Points inducing broadcast traffic from DVB senders. Following the aim of technology independence, the abstraction shall be done in a way that a future additional wireless pointto-multipoint technology can be included without the need of changes at the technology-independent CARMEN mesh functions.

\section{ConcLusions}

The CARMEN project will implement an architecture able to provide operators with a complementary infrastructure able to provide carrier grade services with low operational maintenance, low cost and high flexibility in deployment. This architecture is built with strong bases in heterogeneous technologies, being by this way, able to adapt to different environments and user requirements. In order to use current and future wireless technologies, the CARMEN architecture is based in an abstraction layer which hides the specifics of each technology providing an abstract interface on top of which higher layers can be built. This paper provides a snapshot of the architecture currently under development, showing possible application scenarios and design considerations taken into account in the architecture design phase. The architecture puts emphasis in providing carrier grade services, link and technology abstraction, quality of service and mobility support, all of this integrated in a design oriented to achieve easy deployment and self configuration. In order to be as flexible as possible the architecture also includes continuous monitoring features, which allow self configuration and self healing properties reducing the maintenance cost of the infrastructure. This architecture is conceptually flexible and open, providing clear separation between the technology domain and the administrative domain.

\section{REFERENCES}

[1] "CARMEN: CARrier grade MEsh Networks. ICT project of the EU 7th framework programme," http://www.ict-carmen.eu.

[2] D. Meyer, "BT to create wireless cities," Online Article, May 2006, http://news.zdnet.co.uk/communications/0,1000000085,39269523,00. htm? $\mathrm{r}=1$.

[3] "BT selects Motorola to deploy wireless cities across the UKs," Govermment Technology News Report, Nov. 2006, http://www.govtech.com/ gt/articles/102602.

[4] D. Chieng, T. G. Hodgkinson, A. Ting, R. Rahim, and S. Kawade, "Highlevel capacity performance insights into wireless mesh networking," $B T$ Technology Journal, vol. 25, no. 2, pp. 76-86, Apr. 2007.

[5] N. Bayer, A. Roos, R. P. Karrer, B. Xu, and C. Esteve, "Towards carrier grade wireless mesh networks for broadband access," in 1st Workshop on Operator-Assisted (Wireless Mesh) Community Networks (OpComm 2006), Sept. 2006, pp. 1-10.

[6] "IEEE 802.11 working group on wireless local area networks," http: //www.ieee802.org/11/.

[7] IEEE, "Wireless LAN medium access control (MAC) and physical layer (PHY) specifications: Amendment s: Mesh networking," IEEE P802.11s/D1.07, Sept. 2007.

[8] "IEEE 802.16 working group on broadband wireless access standards," http://www.ieee802.org/16/.

[9] ETSI, "Terrestrial trunked radio (TETRA) - voice plus data $(\mathrm{V}+\mathrm{D})$ part 1: General network design," ETSI ETS 300 392-1, Feb. 1996.

[10] - "Digital video broadcasting: DVB specification for data broadcasting," ETSI EN 301 192, Mar. 2008.

[11] IEEE, "Standard for local and metropolitan area networks: Media independent handover services," IEEE P802.21/D8.0, Dec. 2007. 